\title{
Violent maser events in the circumstellar envelope of the pre-planetary nebula IRAS18276-1431
}

\author{
P. Wolak*, M. Szymczak and A. Bartkiewicz \\ Torun Centre for Astronomy, Faculty of Physics, Astronomy and Informatics, Nicolaus \\ Copernicus University, Poland \\ E-mail: wolakeastro.umk.pl, mszeastro.umk.pl, annan@astro.umk.pl \\ E. Gérard \\ Observatoire de Paris-Meudon, France \\ E-mail: Eric.Gerarddobspm.fr
}

\begin{abstract}
Multi-epoch observations of the pre-planetary nebula IRAS18276-1431 (OH17.7-2.0) have revealed several narrow-band $\mathrm{OH}$ maser flares superimposed on the monotonic decay and rise of the integrated flux density at 1612/1665 and $1667 \mathrm{MHz}$, respectively. The flaring emission was highly polarized and came from well isolated and compact regions of the envelope. Generally the magnetic field prefers the orientation parallel to the axis of the bipolar lobes likely excavated in the envelope by tenuous bipolar winds.
\end{abstract}

12th European VLBI Network Symposium and Users Meeting

7-10 October 2014

Cagliari, Italy

${ }^{*}$ Speaker. 


\section{Introduction}

IRAS18276-1431 is known as a strong $1612 \mathrm{MHz} \mathrm{OH}$ maser source since 1974 [1]. The central star has a spectral type earlier than K5 and effective temperature of 4000-10000 K [8]. It has an optically thick, detached dust shell with a bimodal spectral energy distribution typical for a preplanetary nebula (PPN). The $22 \mathrm{GHz}$ water maser emission was gradually decreasing after 1987 and disappeared in 1991 [5]. The $\mathrm{OH}$ maser envelope shows a significant departure from spherical symmetry. MERLIN observations revealed a magnetic field of $+4.6 \mathrm{mG}$ and $+2.5 \mathrm{mG}$ at 1612 and $1667 \mathrm{MHz}$, respectively, that could be dynamically important for the shaping of the envelope [2]. Observations made in 2010 [7] revealed a weak $\mathrm{SiO}$ maser emission at $43 \mathrm{GHz}$, undetected before [10].

\section{Maser variability}

The $\mathrm{OH}$ maser emission at 1612, 1665 and $1667 \mathrm{MHz}$ was monitored twice a month using the Nançay radio telescope (NRT) from 2002 to 2009 and in 2014. The NRT is a transit instrument (equivalent to a $93 \mathrm{~m}$ parabolic dish). The point-source efficiency was $1.4 \mathrm{~K} \mathrm{Jy}^{-1}$, the system temperature was $35 \mathrm{~K}$. A spectral resolution of $\sim 0.07 \mathrm{~km} \mathrm{~s}^{-1}$ was used. The four Stokes parameters were provided by the system. Bursts of highly polarized $\mathrm{OH}$ maser emission were discovered in the three transition lines. The most prominent one appeared in the red-shifted part of the spectrum part at $\sim 73 \mathrm{~km} \mathrm{~s}^{-1}$ and lasted over $\sim 6$ years (Wolak et al. in prep.). Its integrated flux density increased by a factor $\sim 3$ during 2.5 years (Fig. 1). The integrated flux density of the red-shifted part of the spectrum excluding the bursting feature shows a linear decay similar to the blue-shifted part of the spectrum. Our observations supplemented with archival data confirm a linear decay of the $1612 \mathrm{MHz}$ intensity over $\sim 36$ years. Assuming a constant decrease rate, the $1612 \mathrm{MHz}$ emission will fall below the detection limit before 2030. A monotonic decay and rise of the integrated flux density at 1665 and $1667 \mathrm{MHz}$, respectively, were also noticed (Wolak et al. in prep.). If the maser becomes unsaturated, the decrease may change to the exponential type and the disappearance will be faster.

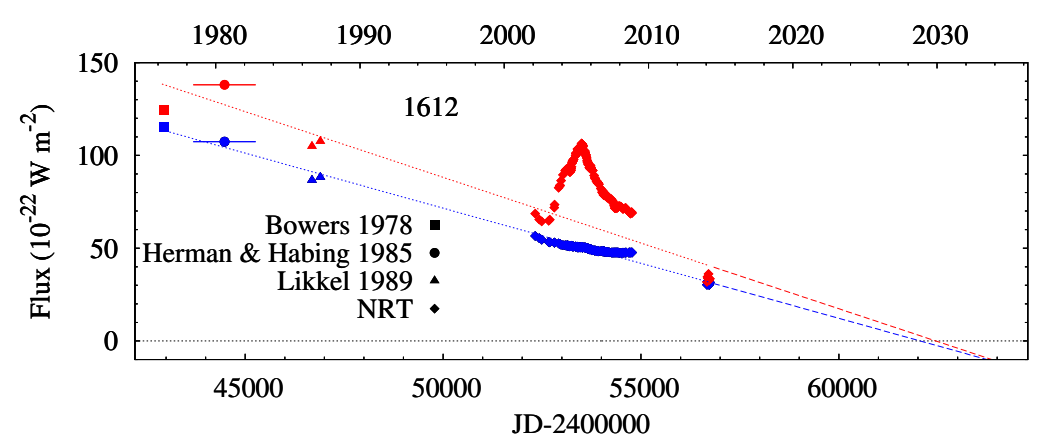

Figure 1: Secular changes of the $\mathrm{OH}$ total flux density at $1612 \mathrm{MHz}$. The red and blue symbols correspond to the total flux density of the red- and blue- shifted parts of the spectrum, respectively. The references to the archival data are given. 
The $22 \mathrm{GHz}$ observations using the Torun $32 \mathrm{~m}$ radio telescope revealed a water maser emission of $25 \mathrm{Jy}$ near $56.3 \mathrm{~km} \mathrm{~s}^{-1}$. The system equivalent flux density was about $480 \mathrm{Jy}$ and the spectral resolution was $0.03 \mathrm{~km} \mathrm{~s}^{-1}$. Thus the emission reappeared after about 20 years. The emission feature has a nearly Gaussian shape and shows a velocity drift from 56.3 to $56.8 \mathrm{~km} \mathrm{~s}^{-1}$ during $\sim 1.5$ year. [11].

\section{Magnetic field structure}

We have mapped the $1612 \mathrm{MHz}$ emission in full polarization at two epochs (2006 and 2007) with MERLIN. The beam size for those observations was $\sim 0 .{ }^{\prime \prime} 45 \times 0 .{ }^{\prime \prime} 25$ and the spectral resolution was $0.18 \mathrm{~km} \mathrm{~s}^{-1}$. The data calibration and reduction were carried out with AIPS using standard procedures for spectral line observations. Archival data from [2] were added for comparison. The spatial distribution of the electric vectors of the linearly polarized maser components globally confirms an ordered magnetic field in the envelope (Fig. 2). The long-lasting burst has occurred in the southern part of the envelope (magnified in Fig.'s 2 inset). In this region the mean polarization position angle $(\chi)$ of the maser components is $-57 \pm 15^{\circ}$. It differs by $-110 \pm 10^{\circ}$ from the mean $\chi$ angle of the remaining $\mathrm{OH}$ components.

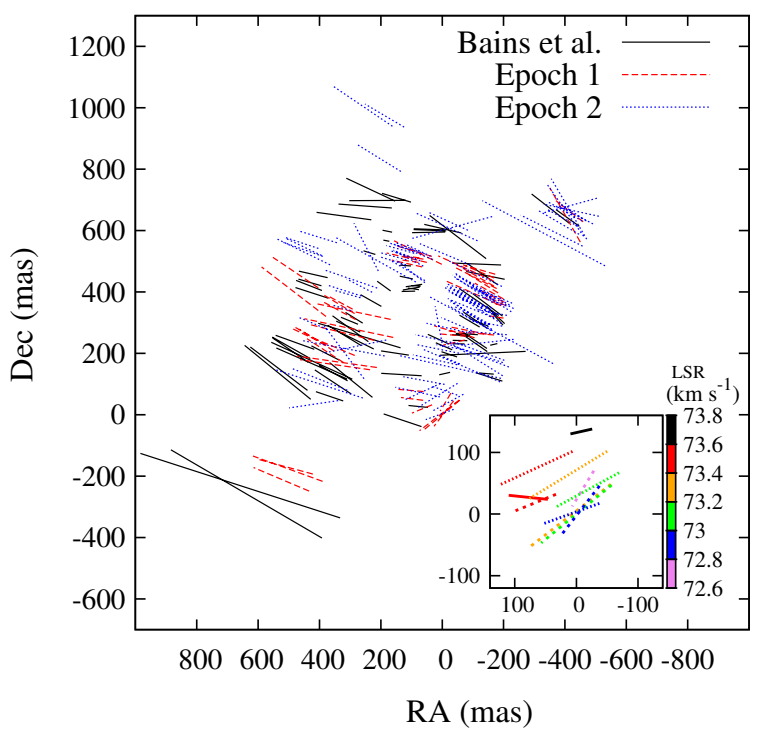

Figure 2: Distribution of the electric field vectors of the linearly polarized components at $1612 \mathrm{MHz}$. The vector lengths are proportional to the degree of linear polarization. Maps from three epochs are presented: 1999 May 18 - black solid lines [2], 2006 May 12 - red dashed lines and 2007 June 05 - blue dotted lines. The inset shows a magnification of the burst region with line colours corresponding to the velocity.

The orientation of the magnetic field vector is determined by the angles $\theta$ and $\chi$, where $\theta$ is the angle between the line of sight and the magnetic field vector. If the ratio of Zeeman splitting to Doppler line width is $>1$ then the degree of linear $m_{\mathrm{L}}$ and circular $m_{\mathrm{C}}$ polarization of the $\sigma$ components are described by equations: $m_{\mathrm{L}}=\frac{\sin ^{2} \theta}{\left(1+\cos ^{2} \theta\right)} 100 \%$ and $m_{\mathrm{C}}=\frac{2 \cos \theta}{\left(1+\cos ^{2} \theta\right)} 100 \%$ [4]. The angle $\theta$ can be calculated from these two equations. Our preliminary study implies that the 
magnetic field vectors in the burst region do not change their orientation randomly but lie in a well defined plane (Wolak et al. in prep.). The size of the burst region is about 25 mas in diameter and is located close to the axis of infrared bipolar lobes reported in [3]. The magnetic field in the burst region is inclined at an angle of $\sim 50^{\circ}$ from the axis of the lobes.

\section{Acknowledgements}

The Nancay Radio Observatory is the Unité Scientifique de Nançay of the Observatoire de Paris, associated with the CNRS. The Nançay Observatory acknowledges the financial support of the Région Centre in France. MERLIN is a UK national facility operated by the University of Manchester on behalf of STFC. This study was partly supported by the Polish National Science Centre through grant 2011/03/B/ST9/00627.

\section{References}

[1] Bowers, P. F. A large-scale OH sky survey at 1612 MHz part. I. The observations, A\&AS, 1978, 31, $127-145$

[2] Bains, I., Gledhill, T. M., Yates, J. A. \& Richards, A. M. S. MERLIN polarimetry of the OH masers in OH17.7-2.0, MNRAS, 2003, 338, 287-302

[3] Sánchez Contreras, C., Le Mignant, D., Sahai, R., Gil de Paz, A. \& Morris, M. Adaptive optics imaging of IRAS 18276-1431: A bipolar preplanetary nebula with circumstellar "searchlight beams" and "arcs", ApJ, 2007, 656, 1150-1166

[4] Elitzur, M. Polarization of astronomical maser radiation. III. Arbitrary Zeeman splitting and anisotropic pumping, ApJ, 1996, 457, 415E

[5] Engels, D. Water vapor masers in stars departing from the AGB, A\&AS, 2002, 338, 252-267

[6] Herman, J. \& Habing, H. J. Time variations and shell sizes of OH masers in late-type stars, A\&AS, $1985,59,523-555$

[7] Kim, J., Cho, S.-H. \& Kim, S. J. Simultaneous observations of $\mathrm{SiO}$ and $\mathrm{H}_{2} \mathrm{O}$ masers toward known stellar $\mathrm{H}_{2} \mathrm{O}$ maser sources, AJ, 2013, 145, 22K

[8] Le Bertre, T., Heydari-Malayeri, M., Epchtein, N., Gouiffes, C. \& Perrier, C. Optical and infrared observations of four suspected proto-planetary objects, A\&A, 1989, 225, 417-431

[9] Likkel, L. OH and $\mathrm{H}_{2} \mathrm{O}$ observations of cold IRAS stars, ApJ, 1989, 344, 350-370

[10] Nyman, L.-A., Hall, P. J. \& Olofsson, H. SiO masers in OH/IR stars, proto-planetary and planetary nebulae, A\&AS, 1998, 127, 185-200

[11] Wolak, P., Szymczak, M., Bartkiewicz, A., et al. Reappearance of $22 \mathrm{GHz}$ water maser in proto-planetary nebula OH17.7-2.0, ATel, 2013, 5211, 1W 